\title{
SGUM-Kurse, Sektion Bewegungsapparat (BWA)
}

Alle offiziellen Ultraschallkurse sind auf der sektionseigenen Homepage www. msus-swiss.ch und www.sgum.ch aufgeführt.

Die SGUM-Kurse (Grund-, Aufbau- und Schlusskurs) finden am 22. und 23 Januar in Bern statt. Details zu den Kursen und Anmeldeinformationen finden Sie unter www.msus-swiss.ch .

Der SGUM-BWA-Refresherkurs findet am 9.9.2015 in Lausanne statt. Das Thema ist die untere Extremität.

- Datum: Mittwoch 9.9.2015

- Kurszeit: 13.00 - $18.00 \mathrm{Uhr}$

- Ort: Congrès Beaulieu Lausanne

- Teilnehmer: Mitglieder SGUM Sektion BWA (Bewegungsapparat) und Inhaber Fähigkeitsausweis SGUM Modul BWA
- Programm

Ab 12.30 Registrierung

13.00-13.30 Vortrag Ultraschall an der Hüfte - R. Ziswiler

13.30-14.00 Exposé échografie du genou - P. Zufferey

14.00-14.30 Vortrag Ultraschall am

Fuss - Ch. Marx

14.30-15.00 Kaffeepause

15.00-15.50 Generalversammlung

SGUM, Sektion BWA

16.00-18.00 praktische Übungen in Kleingruppen, Rotation alle $30 \mathrm{Min}$.

10 Arbeitsplätze 